\title{
Perception of Vibratory Direction on the Back
}

\author{
Astrid M. L. Kappers ${ }^{1,2,3(\bowtie)}{ }_{\mathbb{D}}$, Jill Bay ${ }^{3}$, and Myrthe A. Plaisier ${ }^{1}$ (D) \\ 1 Dynamics and Control, Department of Mechanical Engineering, \\ Eindhoven University of Technology, Eindhoven, The Netherlands \\ \{a.m.l.kappers,m.a.plaisier\}@tue.nl \\ 2 Control Systems Technology, Department of Mechanical Engineering, \\ Eindhoven University of Technology, Eindhoven, The Netherlands \\ 3 Human Technology Interaction, Department of Industrial \\ Engineering \& Innovation Sciences, Eindhoven University of Technology, \\ Eindhoven, The Netherlands
}

\begin{abstract}
In this study, we investigated the accuracy and precision by which vibrotactile directions on the back can be perceived. All direction stimuli consisted of two successive vibrations, the first one always on a centre point on the spine, the second in one of 12 directions equally distributed over a circle. Twelve participants were presented with 144 vibrotactile directions. They were required to match the perceived direction with an arrow they could see and feel on a frontoparallel plane. The results show a clear oblique effect: performance in terms of both precision and accuracy was better with the cardinal directions than with the oblique ones. The results partly reproduce an anisotropy in perceived vertical and horizontal distances observed in other studies.
\end{abstract}

Keywords: Vibrotactile stimulation $\cdot$ Direction perception $\cdot$ Haptic matching.

\section{Introduction}

Vibrotactile displays provide ways to convey information in circumstances where vision or audition are occupied with different tasks or are not available at all. For persons with deafness, blindness or even deafblindness such devices might be helpful in daily tasks such as navigation and communication. In many situations a hands- and head-free device is preferred, and then the back is an obvious choice. Although there certainly has been done some research on the perception of vibrotactile stimulation on the back, the fundamental knowledge at this stage is far from sufficient to design an optimal device. Therefore, the current paper focuses on vibrotactile stimulation on the back, and more in particular, on the perception of direction.

This work was supported by the European Union's Horizon 2020 research and innovation programme under Grant 780814, Project SUITCEYES.

(C) The Author(s) 2020

I. Nisky et al. (Eds.): EuroHaptics 2020, LNCS 12272, pp. 113-121, 2020.

https://doi.org/10.1007/978-3-030-58147-3_13 
There are a few concepts that are of relevance here, and one of these is anisotropy. Weber [9] already found that vertical two-point pressure thresholds on the back are larger than the horizontal thresholds. Although pressure and vibration do not stimulate the same receptors, and thus Weber's observation on pressure thresholds does not necessarily apply to vibratory stimulation, the study by Hoffmann and colleagues [3] points in the same direction: they found that the accuracy of determining the direction of two subsequent vibration stimuli was higher for horizontal than for vertical directions.

Another relevant concept is the "oblique effect". Although this term has been used in many different experimental settings (both visual and haptic), the basic idea is that performance with oblique stimuli is worse than with stimuli oriented in cardinal (i.e. horizontal and vertical) directions. Performance can apply to both accuracy and precision. A task is performed accurately if the setting of the participant is close to the intended physical setting, so this is related to bias or systematic directional error. A task is performed precisely if subsequent measurements consistently lead to the same setting that is not necessarily the correct physical setting. So precision is related to variability or spread. Appelle and Gravetter [1], for example, found that rotating a bar to a specified orientation led to larger variable but not systematic directional errors for oblique orientations in both visual and haptic conditions. Lechelt and Verenka [6] asked participants to match the orientation of a test bar with that of a reference bar in the frontoparallel plane, again in both visual and haptic conditions. They also found much larger variable errors for the oblique orientations, but no directional bias. On the other hand, Kappers [5] reported systematic directional errors when the orientation of a bar had to be matched haptically to a bar at a different location in the horizontal plane. It remains to be seen how representative all these findings are for vibrotactile stimuli on the back.

Finally, it is important to take the difference between simultaneous and successive presentation into account. For pressure stimuli, Weber [9] already observed that the thresholds were smaller if stimuli were presented one after another. Similarly, for vibrotactile stimulation, both Eskilden and colleagues [2] and Novich and Eagleman [7] found better performance with sequential stimulation. Therefore, in the current study, we will only make use of successive stimulation.

In this study, we will investigate the perception of vibrotactile directions on the back. More in particular, we will investigate whether there are biases in the perception of direction, and whether there are differences in spread between the settings for cardinal and oblique directions.

\section{Methods}

\subsection{Participants}

Twelve students ( 7 female, 5 male) of Eindhoven University of Technology participated in this experiment. Their ages ranged between 18 and 23 years. Ten participants were right-handed, two were left-handed (self-report). They were 
unfamiliar with the research questions and the set-up. Before the experiment they gave written informed consent. They received a small financial compensation for participation. The experiments were approved by the Ethical Committee of the Human Technology Interaction group of Eindhoven University of Technology, The Netherlands.

\subsection{Set-Up, Stimuli and Procedure}

Twelve tactors (coin-style ERM vibration motors from Opencircuit, 8 mm diameter) were placed in velcro pockets at every $30^{\circ}$ on a circle with a radius of $110 \mathrm{~mm}$ on the back of an office chair; an identical pocket with tactor was placed in the centre of the circle (see Fig. 1). A distance of $110 \mathrm{~mm}$ is well above the vibrotactile two-point discrimination threshold of $13-60 \mathrm{~mm}$ reported in several papers (e.g. $[4,7,8]$ ), and is about the maximum radius that could be presented on the back. Each trial consisted of a 1-s vibration of the centre tactor, followed by a 1-s break and a 1-s vibration of one of the other 12 tactors. This timing guaranteed that all vibration motors were always easy to distinguish. The vibrations were strong enough to be easily perceived for all locations on the back, but they were not necessarily perceived to be equally strong. During the practice session it was ensured that participants could indeed perceive all motors. Random blocks of the 12 different stimuli were presented 12 times, so the total number of trials per participant was 144 .

The task of the participant was to indicate the direction in which the stimulus was felt by means of rotating an arrow located on a frontoparallel plane at about eye height and within easy reach (see Fig. 1d). They were explicitly instructed to touch the arrowhead with one of their finger tips. The participants put on blurred glasses that still allowed them to see the arrow, but prevented them from reading off the degrees on the protractor. Participants were not informed about the actual directions, nor the number of different directions. The experimenter was able to read off the adjusted orientation with a precision of $1^{\circ}$. Noise-cancelling headphones with white noise and earplugs were used to mask the sound of the vibrators.

Participants were asked to wear thin clothing to guarantee they could feel the vibratory stimulation. At the start of the experiment, the tactors on the chair were covered with a cloth so that the participant remained unware of the actual locations. The participants had to sit down on the chair with their back pressed against the back of the chair. With the help of a line marked on the chair (Fig. 1b), the experimenter made sure that the spine of the participant was aligned with a vertical line through the centre of the circle. The back of the chair was not adjusted in height for the individual participants, but as a difference in body height would result in at most a few $\mathrm{cm}$ difference on the back, the stimulated back areas were still quite similar. The participant was instructed explicitly that s/he should not move to ensure both contact and alignment were kept constant; the experimenter made sure they indeed remained with the back centered on the back rest throughout the experiment. 

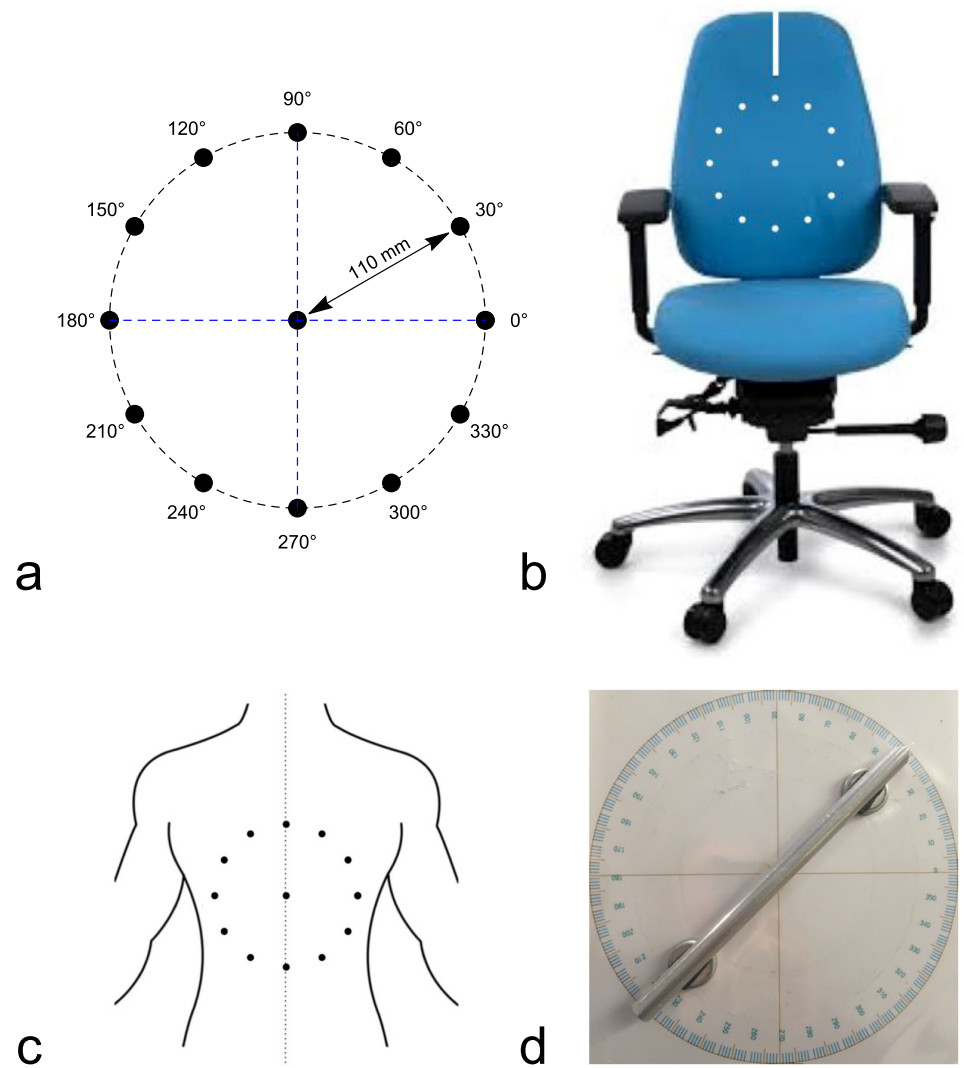

Fig. 1. Set-up. a) Circle with the 12 directions; b) Chair with the positions of the vibration motors (white dots) and the reference line for the spine (white line) indicated; c) Location of the circle of tactors on the back of the participant when seated on the chair; d) Arrow and protractor used to indicate the perceived direction.

The experiment started with a block of 12 different practice trials, after which the participant could ask remaining questions. Neither during the practice trials nor during the actual experiment feedback was given.

\subsection{Data Analysis}

In total there were $1728(12$ participants $\times 144)$ trials. 14 trials were discarded due to technical problems with the tactors. In 17 occasions the matched directions were about $180^{\circ}$ off. This could either be due to a misperception of the participant or ignorance of the arrowhead. As the latter explanation seems much more likely than the former (some participants indeed confessed that they sometimes forgot to attend to the arrowhead), we decided to correct these cases. Finally, there were 16 clear outliers (leaving out such points led to a reduction 
in the standard deviation by at least a factor 2 , but often much more). As these would have enormous effects on the standard deviations without being representative, it was decided to discard these 16 trials (less dan 1\%).

For all analyses, we first computed mean and standard deviations per participant and per direction. Subsequently, we computed means and standard deviations over participants but per direction. We also compared results for cardinal $\left(0^{\circ}, 90^{\circ}, 180^{\circ}\right.$ and $\left.270^{\circ}\right)$ and oblique (all other) directions.

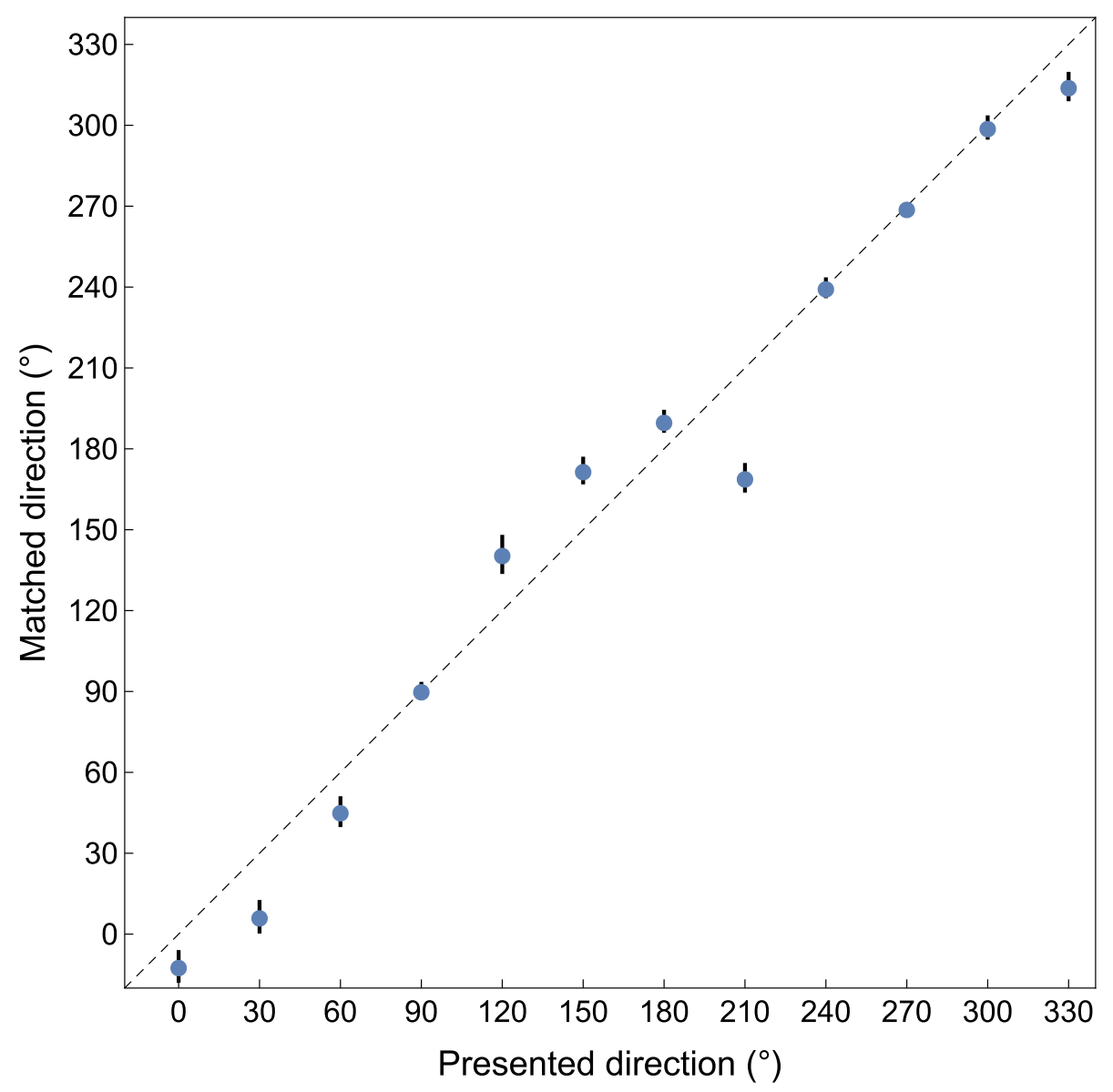

Fig. 2. Matched directions as a function of presented directions averaged over all participants. The error bars indicate standard errors of the mean and the dashed line the unity line.

\section{Results}

In Fig. 2 the matched directions are shown as a function of the presented directions. The error bars indicate standard errors over the averages of participants. 


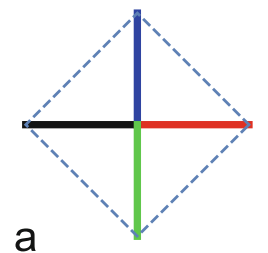

b

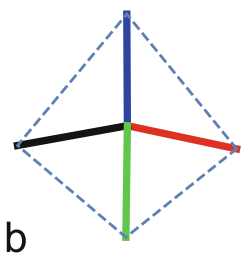

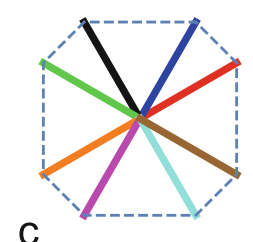

C

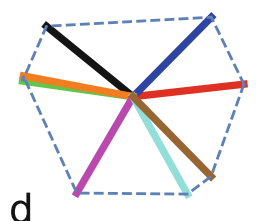

Fig. 3. Graphical representation of the deviations shown in Fig. 2. a) Presented cardinal directions; b) Matched cardinal directions; c) Presented oblique orientations; d) Matched oblique orientations. The same colours in a and b, and in c and d indicate pairs of presented and matched directions. (Color figure online)

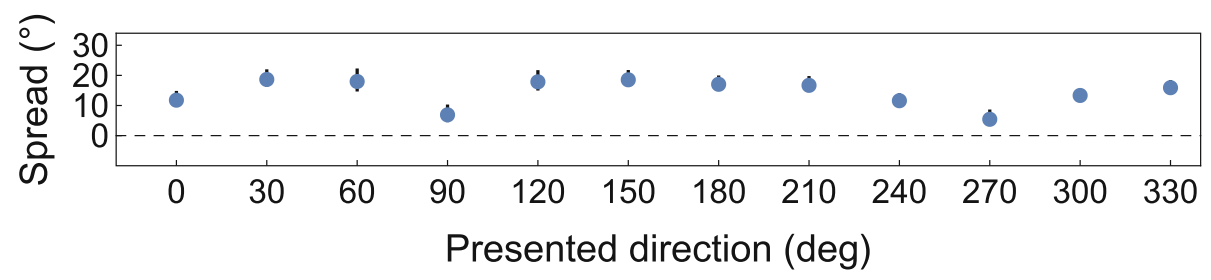

Fig. 4. Standard deviations (spread) averaged over participants as a function of presented directions. The error bars (these are so small that they are hardly visible) indicate standard errors of the mean.

It can be seen that there is quite some variation: some directions are clearly underestimated, whereas some other directions are overestimated. A graphical representation of these mismatches is shown in Fig. 3 for the cardinal and oblique directions separately. In Fig. 3a and b it can be seen that the vertical directions are matched correctly, whereas the horizontal directions point somewhat downward. The upward oblique orientations are all adjusted more horizontally, whereas the downward oblique directions do not show a clear pattern (Fig. 3c and d).

In Fig. 4 the standard deviations (spread) averaged over participants is shown. These values give an indication about how precise the participants are in their matching performance. It can be seen that especially the spread of the two vertical directions $\left(90^{\circ}\right.$ and $\left.270^{\circ}\right)$ is quite small.

One of the research questions is whether there are differences in performance between cardinal and oblique direction as has been found in other studies not using vibrotactile stimuli and not presented on the back (e.g. $[1,5,6])$. To investigate this, we need to look at the absolute values of the mean deviations per participant, because signed values might average out over the various directions (see Fig. 2). In Fig. 5a we show the absolute mean deviations averaged over participants for both the cardinal $(M=9.4, S D=6.2)$ and oblique $(M=20.2, S D$ $=5.5$ ) directions. It can clearly be seen that the values of the oblique directions are higher than those of the cardinal directions. A paired $t$-test shows that this difference is highly significant: $t(11)=5.5, p<0.0002$. In Fig. 5b we compare the 

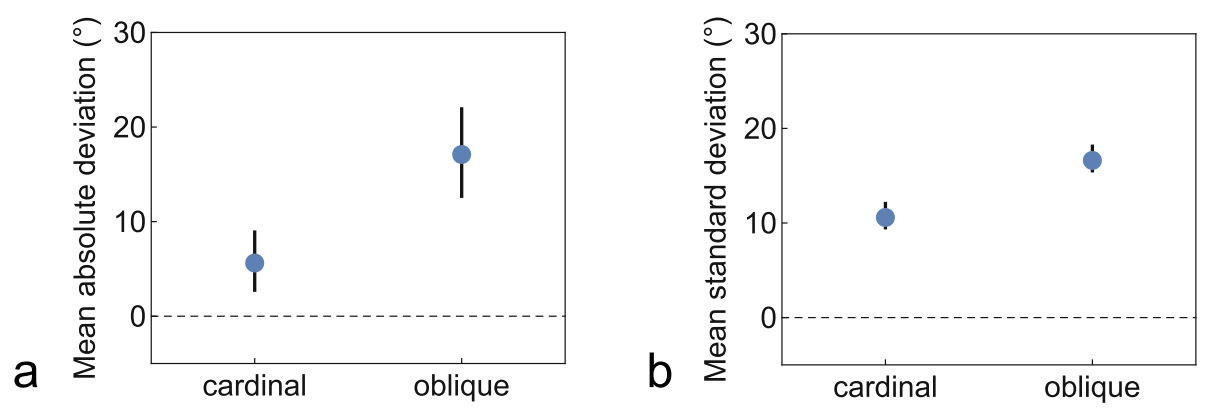

Fig. 5. Comparison of performance on cardinal and oblique directions. a) Absolute mean deviations averaged over participants for both the cardinal and oblique directions; b) Standard deviations in the cardinal and oblique directions averaged over participants. The error bars indicate standard errors of the mean.

spread of the deviations in the cardinal $(M=10.8, S D=4.4)$ and oblique $(M$ $=16.8, S D=4.5)$ directions. Also this difference is significant: $t(11)=4.2, p<$ 0.002 .

\section{Discussion and Conclusions}

The aim of this study was to investigate the perception of vibrotactile directions presented on the back. The results show that the participants were well able to do this task, although they made some systematic directional errors. In Figs. 2 and 3 , it can be seen that vertical directions $\left(90^{\circ}\right.$ and $\left.270^{\circ}\right)$ were perceived veridical and in Fig. 4 it can be seen that also the variable errors for these directions were small. As the tactors used to generate these directions were all located on the spine of the participants, it is likely that perception was helped by the spine serving as anchor point. Other studies on vibrotactile perception also mention improved performance on or near the spine (e.g. [3,8]).

For the horizontal directions (especially $0^{\circ}$ ) the directional and variable errors are also relatively small, although perception is not veridical. Both horizontal directions are perceived as somewhat downward. Interestingly, Weber [9] already observed a somewhat oblique orientation for a two-point pressure threshold measurement on the back, albeit that this seems a rather informal observation without a mention of the actual direction. Novich and Eagleman [7] did not find confusions of their horizontal vibrotactile stimuli with oblique stimuli. However, in their 8-alternatives forced-choice experiment participants had the choice of 4 cardinal directions and 4 diagonal directions. Confusing horizontal with oblique would imply a misperception of $45^{\circ}$ which is probably a too large difference.

The oblique directions caused both larger directional errors (biases) and larger variability of the errors than the cardinal directions. The type of deviations can best be appreciated in Fig. 3d. Especially the upward oblique directions appear to be perceived as closer to horizontal. A similar finding was reported 
by Novich and Eagleman [7]. Using somewhat smaller distances, they showed that especially the upward oblique directions were perceived as horizontal. Also relevant here are the results of the study by Hoffmann et al. [3] who found an anisotropy in horizontal and vertical acuity: their vertical distances were perceived as smaller than the horizontal distances. In our experiment, such an anisotropy would lead to oblique directions being perceived towards the horizontal and that is what we found for 5 out of the 8 oblique directions.

This study provides insights into how accurate and precise vibrotactile directions can be perceived. This is useful information for the design of vibrotactile devices intended to convey information to the users. In the current study, the first active tactor was always located on the spine. As the spine may have served as an anchor point, it remains a question whether the results are representative for a similar off-centre presentation of directions.

\section{References}

1. Appelle, S., Gravetter, F.: Effect of modality-specific experience on visual and haptic judgment of orientation. Perception 14(6), 763-773 (1985). https://doi.org/10. 1068/p140763

2. Eskildsen, P., Morris, A., Collins, C.C., Bach-y-Rita, P.: Simultaneous and successive cutaneous two-point thresholds for vibration. Psychonomic Sci. 14(4), 146-147 (1969). https://doi.org/10.3758/BF03332755

3. Hoffmann, R., Valgeirsdóttir, V.V., Jóhannesson, Ó.I., Unnthorsson, R., Kristjánsson, Á.: Measuring relative vibrotactile spatial acuity: effects of tactor type, anchor points and tactile anisotropy. Exp. Brain Res. 236(12), 3405-3416 (2018). https://doi.org/10.1007/s00221-018-5387-z

4. Jóhannesson, Ó.I., Hoffmann, R., Valgeirsdóttir, V.V., Unnpórsson, R., Moldoveanu, A., Kristjánsson, Á.: Relative vibrotactile spatial acuity of the torso. Exp. Brain Res. 235(11), 3505-3515 (2017). https://doi.org/10.1007/s00221-017-5073-6

5. Kappers, A.M.L.: Large systematic deviations in a bimanual parallelity task: further analysis of contributing factors. Acta Psychologica 114(2), 131-145 (2003). https:// doi.org/10.1016/S0001-6918(03)00063-5

6. Lechelt, E.C., Verenka, A.: Spatial anisotropy in intramodal and cross-modal judgments of stimulus orientation: the stability of the oblique effect. Perception 9(5), 581-589 (1980). https://doi.org/10.1068/p090581

7. Novich, S.D., Eagleman, D.M.: Using space and time to encode vibrotactile information: toward an estimate of the skin's achievable throughput. Exp. Brain Res. 233(10), 2777-2788 (2015). https://doi.org/10.1007/s00221-015-4346-1

8. Van Erp, J.B.F.: Vibrotactile spatial acuity on the torso: effects of location and timing parameters. In: First Joint Eurohaptics Conference and Symposium on Haptic Interfaces for Virtual Environment and Teleoperator Systems. World Haptics Conference. pp. 80-85 (2005). https://doi.org/10.1109/WHC.2005.144

9. Weber, E.H.: E.H. Weber on the Tactile Senses. Ross, H.E., Murray, D.J. (eds.) Erlbaum (UK), Taylor \& Francis (1834/1986) 
Open Access This chapter is licensed under the terms of the Creative Commons Attribution 4.0 International License (http://creativecommons.org/licenses/by/4.0/), which permits use, sharing, adaptation, distribution and reproduction in any medium or format, as long as you give appropriate credit to the original author(s) and the source, provide a link to the Creative Commons license and indicate if changes were made.

The images or other third party material in this chapter are included in the chapter's Creative Commons license, unless indicated otherwise in a credit line to the material. If material is not included in the chapter's Creative Commons license and your intended use is not permitted by statutory regulation or exceeds the permitted use, you will need to obtain permission directly from the copyright holder.

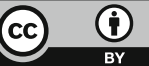

